\title{
LINEAR AND NONLINEAR FRACTIONAL HEREDITARY CONSTITUTIVE LAWS OF ASPHALT MIXTURES
}

\author{
Gaetano DI MINO ${ }^{\mathrm{a}}$, Gordon AIREY ${ }^{\mathrm{b}}$, Mario DI PAOLA ${ }^{\mathrm{a}}$, Francesco Paolo PINNOLA ${ }^{\mathrm{a}}$, \\ Giacomo D'ANGELO ${ }^{a}$, Davide LO PRESTI ${ }^{\mathrm{b}}$ \\ ${ }^{a}$ Dipartimento di Ingegneria Civile, Ambientale, Aerospaziale e dei Materiali (DICAM), \\ Università degli Studi di Palermo, Viale delle Scienze, Ed. 8, 90128 Palermo, Italy \\ ${ }^{b}$ Nottingham Centre for Pavement Engineering, University of Nottingham, UK
}

Received 22 Oct 2013; accepted 21 Nov 2013

\begin{abstract}
The aim of this paper is to propose a fractional viscoelastic and viscoplastic model of asphalt mixtures using experimental data of several tests such as creep and creep recovery performed at different temperatures and at different stress levels. From a best fitting procedure it is shown that both the creep one and recovery curve follow a power law model. It is shown that the suitable model for asphalt mixtures is a dashpot and a fractional element arranged in series. The proposed model is also available outside of the linear domain but in this case the parameters of the model depend on the stress level.
\end{abstract}

Keywords: fractional calculus, asphalt mixture, viscoelasticity, viscoplasticity, rheology, mechanical models, creep test.

\section{Introduction}

The mechanical behaviour of asphalt mixtures is traditionally predicted by classical Kelvin-Voigt or Maxwell elements that are composed of spring and dashpot arranged in parallel or in series, respectively. In order to fit experimental data of asphalt mixture other more sophisticated models like Zener, Burger (Liu, You 2009) or other arrangements of elementary units of springs and dashpots have been used (Werkmeister et al. 2013). Whatever the number and the arrangement of elementary units are, the kernel in the Boltzmann superposition principle has an exponential kernel. On the other hand Nutting (1921) observed that the creep or the relaxation test performed on any real material follow a power law kernel instead of an exponential one. Based on this observation the constitutive law of any real material, including rubber, glass, asphalt mixture, is ruled by a fractional operator (Koeller 1984; Bagley, Torvik 1983, 1986; Slonimsky 1967; Smit, de Vrie 1970; Soczkiewicz 2002; Di Paola et al. 2011). The characterization of the real material by means of fractional derivative and integrals of real order produces strong variations on the response with respect to the characterization involving derivatives (or integrals) of integer order. While the integer order derivative involves the knowledge of the state of the system at one or more previous instants, when the fractional operator appears the entire past history (hereditary materials) gives information at the given time instant (long tail memory).
From recent literature on the subject for bitumen and asphalt mixture based upon fractional constitutive laws the readers may be referred to Di Paola et al. (2009); Celauro et al. (2012); Stastna and Zanzotto 1994, 1996; Oeser et al. (2008). In previous papers the mechanical model was composed of a fractional element alone (usually termed as springpot in literature) or arranged in parallel with a spring. That is the Kelvin-Voigt fractional element.

In this paper, based upon experimental tests performed on asphalt mixtures at several stress levels and various temperatures, a quite different model is proposed. It is a springpot in series with a dashpot element. With this choice the creep function, obtained by a best fitting procedure, gives impressive match between experimental results and theoretical ones. This result is obtained for any temperature $T$ and for any stress intensity. As experimental creep tests were performed at high stress levels, the nonlinear behaviour was found. This is confirmed by the fact that the characteristic parameters of both the dashpot and the springpot are different for different stress levels. This renders the constitutive law nonlinear in the sense that the characteristic coefficients of the dashpot and the springpot will depend not only on the temperature, but also on the stress level. The nonlinearity of response in terms of the strain also depends on the signum of the derivative of the stress $(\operatorname{sign}(\dot{\sigma}))$. It is shown that with this important modification on the dependence of the parameters from a

Corresponding author: Gaetano Di Mino

E-mail: gaetano.dimino@unipa.it 
physical point of view leads to a residual strain how it happens on the plasticity. Then at high stress level the behaviour of the asphalt mixture is viscoplastic.

It is shown that the proposed model is fully available for monotonic stress history. For non-monotonic ones other experimental creep recovery tests are necessary for the complete definition of the material at hands.

\section{Fractional linear viscoelastic model}

The constitutive law of any linear viscoelastic material may be obtained by starting from the creep test. Such a test is performed by putting a constant load at time $t=0$ and by measuring the corresponding strain in time. Then the creep $J(t)$ function is the strain for the stress history $U(t), U(t)$ being the unit step function.

Once $J(t)$ is known from the experimental test, the Boltzmann superposition principle gives the strain history $\varepsilon(t)$ for any stress distribution $\sigma(t)$ in the form:

$$
\varepsilon(t)=\int_{0}^{t} J(t-\tau) \dot{\sigma}(\tau) d \tau
$$

Eqn (1) is valid for $\sigma(0)=0$. If, $\sigma(0) \neq 0$ then in Eqn (1) the term $J(t) \sigma(0)$ has to be added.

Another function useful in the viscoelastic theory is the relaxation function, in the following denoted as $G(t)$. This function is the stress history for an imposed strain history $U(t)$. Using again the Boltzmann superposition principle we get:

$$
\sigma(t)=\int_{0}^{t} G(t-\tau) \dot{\varepsilon}(\tau) d \tau
$$

If the initial condition $\varepsilon(0) \neq 0$, then the term $G(t) \varepsilon(0)$ has to be added in Eqn (2). By making the Laplace Transform of Eqns (1) and (2) and equating the ratio between the Laplace Transform of $\sigma(t)$ and $\varepsilon(t)$, the following fundamental relation between the Laplace Transform of $J(t)$ and $G(t)$ is obtained as:

$$
\hat{G}(s) \hat{J}(s)=\frac{1}{s^{2}},
$$

where $\hat{J}(s)$ and $\hat{G}(s)$ are the Laplace Transform of $J(t)$ and $G(t)$ respectively and $s$ is the Laplace parameter (Christensen 1982; Flügge 1975).

Experimental creep tests on real materials like polymers, rubber, bitumen and so on are well fitted by a power law of the type:

$$
J(t)=\frac{t^{\alpha}}{C_{\alpha} \Gamma(1+\alpha)},
$$

where $\Gamma(\cdot)$ is the Euler Gamma function and $\alpha, C_{\alpha}$ are parameters obtained by best fitting on experimental data. By using Eqn (3), the corresponding relaxation function is readily found in the form:

$$
G(t)=\frac{C_{\alpha} t^{-\alpha}}{\Gamma(1-\alpha)} .
$$

By inserting Eqns (4) and (5) into (1) and (2), respectively, we get:

$$
\begin{aligned}
\varepsilon(t)= & \frac{1}{C_{\alpha} \Gamma(1+\alpha)} \int_{0}^{t}(t-\tau)^{\alpha} \dot{\sigma}(\tau) \mathrm{d} \tau= \\
& \frac{1}{C_{\alpha} \Gamma(1+\alpha)}\left[(t-\tau)^{\alpha} \sigma(\tau) \mathrm{d} \tau\right]_{0}^{t}+ \\
& \frac{1}{C_{\alpha} \Gamma(1+\alpha)} \int_{0}^{t}(t-\tau)^{\alpha-1} \sigma(\tau) \mathrm{d} \tau=\frac{1}{C_{\alpha}}\left(I_{0^{+}}^{\alpha} \sigma\right)(t) ; \\
\sigma(t)= & \frac{C_{\alpha}}{\Gamma(1-\alpha)} \int_{0}^{t}(t-\tau)^{-\alpha} \dot{\varepsilon}(\tau) \mathrm{d} \tau=C_{\alpha}\left({ }^{c} D_{0^{+}}^{\alpha} \varepsilon\right)(t) .
\end{aligned}
$$

Eqn (6) is valid provided $\sigma(0)=0$. In Eqn (6) the symbol $\left(I_{0^{+}}^{\alpha} \sigma\right)(t)$ is the Riemann-Liouville fractional integral and in Eqn (7) $\left({ }^{c} D_{0^{+}}^{\alpha} \varepsilon\right)(t)$ is the Caputo's fractional derivative. Eqns (6) and (7) are the constitutive laws of the viscoelastic material at hand. It is to be emphasized that $0 \leq \alpha \leq 1$. In particular if $\alpha=0$ then the material is purely elastic, if $\alpha=1$ then the material is a pure fluid and $C_{\alpha}$ is its viscosity coefficient. More detailed discussions on this point may be found in Di Paola and Zingales (2012); Di Paola et al. (2013).

More complex behaviour may be obtained by enriching the creep function by additional terms (Podlubny 1999; Mainardi 2010; Di Paola et al. 2013; Grzesikiewicz et al. 2013; Zbiciak 2013). Eqns (6) and (7) are the starting points to characterize the viscoelastic properties of materials.

\section{Experimental investigation on asphalt mixtures}

In order to investigate the viscoelastic properties of the asphalt mixtures a number of uniaxial creep compression tests have been carried out at the laboratory of University of Nottingham. The tests have been performed according to UNI EN 12697 (2005), on two typical bituminous mixtures (British Standards Institution 2003).

The first mixture is Hot Rolled Asphalt (HRA), generally used as wearing course in flexible pavements, which is characterized by a gap-graded mixture with very little medium-sized aggregate. Figure 1 shows a typical aggregate grading curve and a cross section of a HRA sample.

The second mixture is Dense Bitumen Macadam (DBM), generally used as a base layer in flexible pavements, which is characterized by a continuously graded mixture. A typical grading and an idealised section through this mixture are shown in Figure 2.

In order to study only the effect of different aggregate structures on the deformation behaviour of mixtures, the same bitumen $\left(70 / 100\right.$ pen; softening point at $\left.45^{\circ} \mathrm{C}\right)$ was used in both mixtures. A total of 28 specimens were prepared and tested. Uniaxial creep compression tests were performed at three temperatures $(10,20$ and $40{ }^{\circ} \mathrm{C}$ ) and various stress levels depending on the test temperature. 


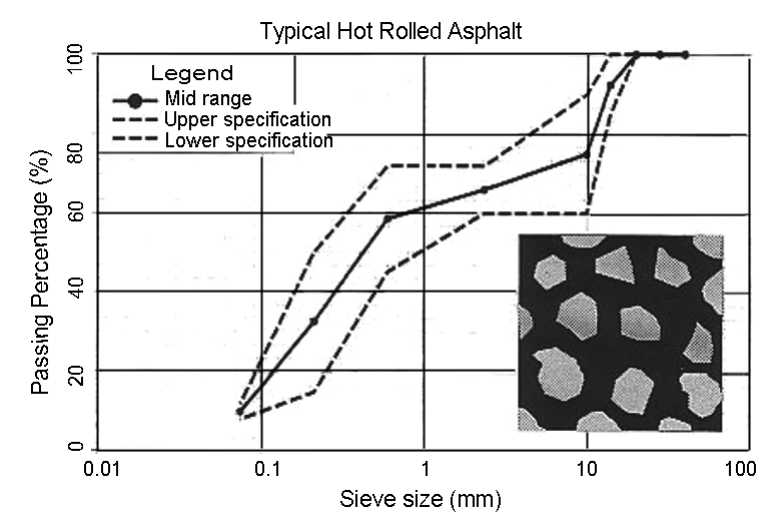

Fig. 1. A typical grading and cross section of a HRA30/14

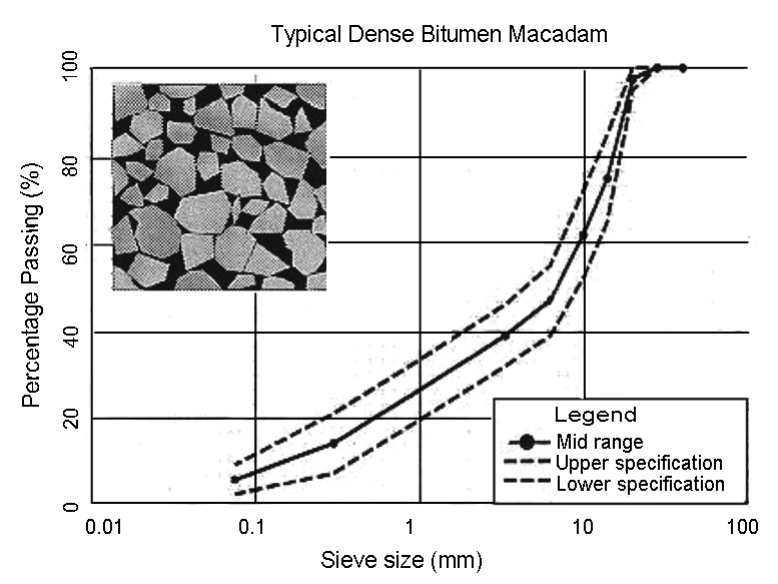

Fig. 2. A typical grading and cross section of a $20 \mathrm{~mm} \mathrm{DBM}$

\section{Results and best fitting procedure}

In order to obtain the parameters of the model, a best fitting procedure was implemented using Wolfram Mathematica $9.0^{\circledR}$ software with the experimental data regarding creep and creep recovery tests.

Various candidate shapes of power law have been analysed. The optimum shape that has been obtained for both HRA and DBM is given as follows:

$$
J(t)=U(t)\left(\frac{t}{c}+\frac{t^{\alpha}}{C_{\alpha} \alpha \Gamma(\alpha)}\right),
$$

where $c$ is the viscosity coefficient, $C_{\alpha}$ and $\alpha$ are parameters related to temperature, stiffness and viscosity.

In all cases Eqn (8) fitted the experimental data very well.

The mechanical equivalent of creep curve described in Eqn (8) is a dashpot (term $t / c$ ) and a so called springpot (term $t^{\alpha} / C_{\alpha} \alpha \Gamma(\alpha)$ ) in series as shown in Figure 3. The results are contrasted with the experimental creep tests. Figures 4 and 5 summarize the results obtained by the best fitting procedure whose relevant parameters are reported in Table 1 (other Figures and Tables can be downloaded from the following link:

https://www.dropbox.com/sh/ju3263gzsui2znx/oAQfPPtxfI ).
Once the creep law is fixed then as soon as we assume $c, C_{\alpha}, \alpha$ independent of the stress level the linear law of viscoelastic asphalt mixture is given as:

$$
\varepsilon(t)=\frac{1}{c}\left(I_{0^{+}}^{1} \sigma\right)(t)+\frac{1}{C_{\alpha}}\left(I_{0^{+}}^{\alpha} \sigma\right)(t) .
$$

Eqn (9) states the strain history due to the assigned stress history $\sigma(t)$ is composed by two terms: a purely viscous part as the first term and a fractional elastic-viscous term ruled by the fractional operator. The latter is ruled by the order $\alpha$ of the fractional integral. If $\alpha=0$ then the second term is purely elastic and Eqn (9) is a classical Maxwell element. If $\alpha=1$ Eqn (9) reverts into two dashpots arranged in series. When $0 \leq \alpha \leq 1$ the mechanical model is that represented in Figure 3, where the fractional term is a springpot that has an intermediate behaviour between elastic and viscous.

It has to be emphasized that if $c \rightarrow \infty$, then $J(t)=$ $\frac{t^{\alpha}}{C_{\alpha} \Gamma(1+\alpha)}$ is a purely viscoelastic term and is the wellknown Scott-Blair model. In Figures 4-9 in dashed line the results obtained by best fitting procedure are reported, showing that the experimental date are better fitted with Eqn (8).

Once the constitutive laws have been derived from best fitting procedure based on experimental data some considerations on the physical properties of the material can be drawn, in particular on the role played by the parameters $c, C_{\alpha}$ and $\alpha$.

As the temperature increases the value of $c$ highly decreases. This means that the deformation is mainly related to the fluid phase. As in fact the smaller $C_{\alpha}$ the higher the deformation of the dashpot element represented in Figure 3 is.

As the temperature increases the values of $C_{\alpha}$ and $\alpha$ moderately decrease. That is the relative weight of the viscoelastic component (springpot in Fig. 3) of the asphalt mixture, is less sensitive to the temperature. In any cases since the smaller $C_{\alpha}$ the higher the deformation is, we can state that also the viscoelastic component gives an increasing value to the total deformation (as we expect). On the other hand $\alpha$ decreases as the temperature increases.

As a conclusion we can state that as the temperature increases the viscous phase, coming in part from dashpot and in part from the fractional element, prevails on the elastic component.

In Table 1 the symbol $t_{1}$ is the time at which the assigned stress level is reached by the test machine.

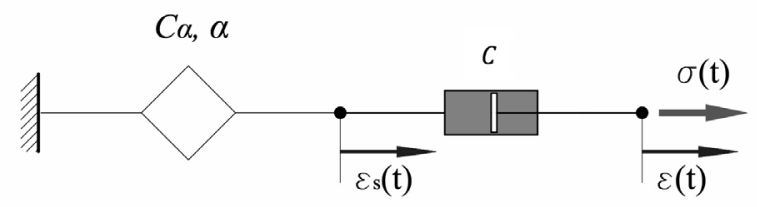

Fig. 3. Springpot element in series with a dashpot 


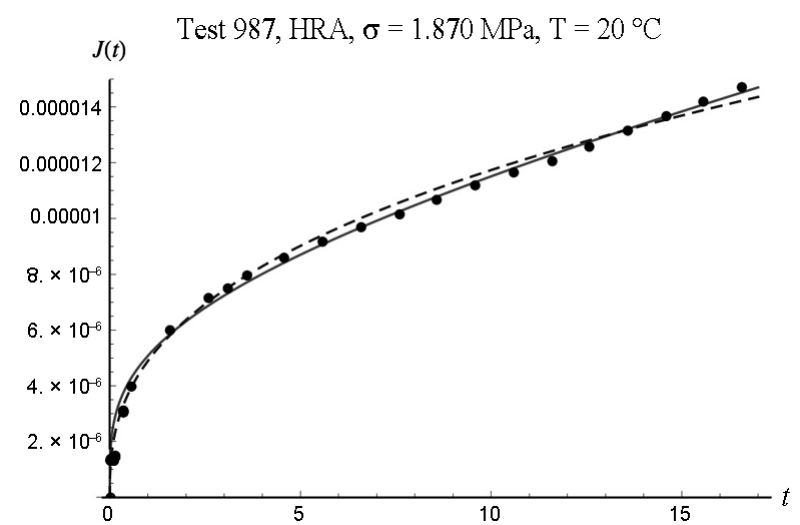

Fig. 4. HRA Experimental data (dots) contrasted with bestfitting curves of the proposed model (continuous line) and the Scott-Blair model (dashed line), $20^{\circ} \mathrm{C}$

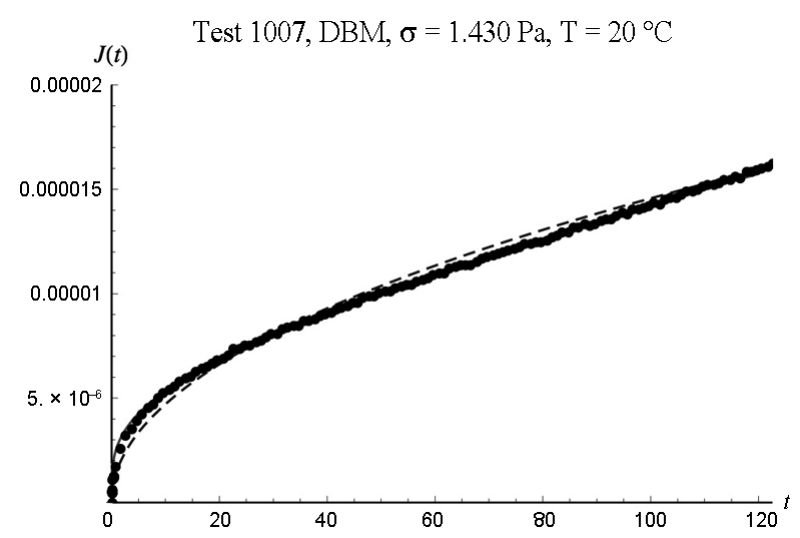

Fig. 5. DBM Experimental data (dots) contrasted with bestfitting curves of the proposed model (continuous line) and the Scott-Blair model (dashed line), $20^{\circ} \mathrm{C}$

Table 1. Best fitting parameters of creep tests 987 (HRA) and 1007 (DBM)

\begin{tabular}{|c|c|c|c|c|c|c|c|c|}
\hline \multirow{3}{*}{ Test } & \multirow{3}{*}{ A.M. } & \multirow{2}{*}{$\begin{array}{c}\text { Air } \\
\text { Voids }\end{array}$} & \multirow{2}{*}{ Stress } & \multirow{2}{*}{$\mathrm{t} 1$} & \multirow{2}{*}{$\mathrm{T}$} & \multicolumn{3}{|c|}{$\begin{array}{l}\text { Fractional proposed } \\
\text { model }\end{array}$} \\
\hline & & & & & & $c^{*} 10^{-3}$ & $\mathrm{C} \alpha^{*} 10^{-3}$ & $\alpha$ \\
\hline & & {$[\%]$} & {$[\mathrm{kPa}]$} & {$[\mathrm{s}]$} & {$\left[{ }^{\circ} \mathrm{C}\right]$} & [Mpa s] & $\begin{array}{c}{[\mathrm{Mpa}} \\
\left.\mathrm{s}^{\wedge} \alpha\right]\end{array}$ & {$[-]$} \\
\hline 987 & HRA & 4.6 & 1870 & 0.126 & 20 & 3800 & 230.0 & 0.266 \\
\hline 1007 & DBM & 5 & 1430 & 0.097 & 20 & 18500 & 425.0 & 0.265 \\
\hline
\end{tabular}

From data obtained from all creep tests some considerations can be drawn: 1) for fixed stress level and temperature the best fitting parameters showed very close values to each other; 2) all $c, C_{\alpha}, \alpha$ parameters are very sensitive to the temperature variations; 3 ) other test conditions being equal the DBM's $C_{\alpha}$ was higher than HRA's one, as confirmed from dynamic tests carried out at Nottingham.

In the next section a detailed description on the nonlinear behaviour of the asphalt mixture will be presented.

\section{Nonlinear behaviour}

It is widely recognized that linear viscoelasticity holds at a low stress level. In this range parameters $c, C_{\alpha}, \alpha$ remain constant, whereas as soon as the stress level increases in creep tests (at fixed temperature), the various parameters exhibit variations and the Boltzmann superposition principle fails. Detailed discussion on this point may be found in Airey et al. (2003); Airey and Rahimzadeh (2004); Findley and Onaran (1976). For the particular case of asphalt mixture presented here the linearity strain range has to be of order $10^{-4}$ (Airey 2004), that is much lower than the strain levels corresponding to the laboratory tests. This is due to the fact that the goal of these tests was the study of steady-state deformation behaviour (Taherkhania 2011) or to evaluate mechanical parameters of the Burger generalized model (Huang 2004). From the experimental tests reported in Tables 1-2, we may affirm that the coefficients to describe the power law of the mixtures under study depend on temperature as well stress intensity. Because of the dependence of the stress level as first attempt we may suppose that the three parameters $c, C_{\alpha}, \alpha$ depend on the stress intensity and on the temperature, that is:

$$
c=c(T, \sigma) ; C_{\alpha}=C_{\alpha}(T, \sigma) ; \alpha=\alpha(T, \sigma) .
$$

From the data exploited in Tables 1-2, we may suppose that for fixed value of $T$, the relevant parameter may be expressed in the form:

$$
\begin{aligned}
& \frac{1}{c}=a_{c}+b_{c} \sigma^{\beta} ; \\
& C_{\alpha}=a_{C_{\alpha}}+b_{C_{\alpha}} \sigma^{\gamma} ; \\
& \alpha=a_{\alpha}+b_{\alpha} \sigma^{\eta},
\end{aligned}
$$

where the various parameters, $a_{\mathrm{c}}, b_{\mathrm{c}}, a_{C_{\alpha}}, b_{C_{\alpha}}, a_{\alpha}, b_{\alpha}, \beta$, $\gamma, \eta$, are determined by the creep test at the various stress intensities. The limitation on $\beta, \gamma, \eta$ is $\beta, \gamma, \eta>1$. This limitation is due to the fact that at low stress level the material will behave linearly and then the various parameters will remain constant in proximity of $\sigma=0$. It follows that the slope of the coefficients in Eqn (10) has to be zero as $\sigma \rightarrow 0$, that is:

$\left.\frac{\partial c(T, \sigma)}{\partial \sigma}\right|_{\sigma=0}=0 ;\left.\frac{\partial C_{\alpha}(T, \sigma)}{\partial \sigma}\right|_{\sigma=0}=0 ;\left.\frac{\partial_{\alpha}(T, \sigma)}{\partial \sigma}\right|_{\sigma=0}=0$.

$(12 \mathrm{a}, \mathrm{b}, \mathrm{c})$

Conditions (12) are satisfied only if $\beta, \gamma, \eta>1$. In Figure 6 the only trend of $1 / c$ for HRA is reported (all data are contained in $*$ ). The other limitations on the various coefficients are $c>0, C_{\alpha}<0,0 \leq \alpha \leq 1$.

In Tables 2 and 3 the various coefficients in Eqn (11a,b) are reported for both HRA and DBM.

If we suppose that $c, C_{\alpha}, \alpha$ at a given temperature depend only on the stress amplitude $\sigma_{0}$, then Eqn (9) has to be modified in the form: 


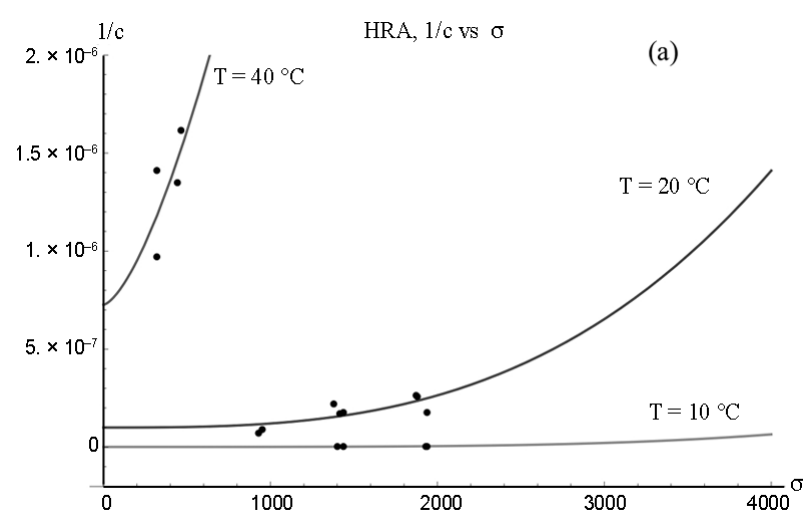

Fig. 6. Experimental trend of $1 / c$ parameter at different temperatures (HRA)

Table 2. Coefficients in Eqn (12a,b) for HRA

\begin{tabular}{ccccc}
\hline \multirow{2}{*}{ HRA } & \multicolumn{4}{c}{$\mathrm{T}\left[{ }^{\circ} \mathrm{C}\right]$} \\
\cline { 2 - 5 } & & 10 & 20 & 40 \\
\hline \multirow{3}{*}{$1 / c$} & $\mathrm{a}_{c}$ & $1.36 \mathrm{E}-09$ & $1.00 \mathrm{E}-07$ & $7.27 \mathrm{E}-07$ \\
\cline { 2 - 5 } & $\mathrm{b}_{c}$ & $2.49 \mathrm{E}-22$ & $2.05 \mathrm{E}-17$ & $7.91 \mathrm{E}-11$ \\
\cline { 2 - 5 } & $\beta$ & 4 & 3 & 1.5 \\
\hline \multirow{3}{*}{$\mathrm{C}_{\alpha}$} & $\mathrm{a}_{c}$ & 247.7 & 170.6 & 27.3 \\
\cline { 2 - 5 } & $\mathrm{b}_{c}$ & $6.65 \mathrm{E}-10$ & $1.15 \mathrm{E}-08$ & $9.81 \mathrm{E}-03$ \\
\cline { 2 - 5 } & $\gamma$ & 3.5 & 3 & 1.3 \\
\hline
\end{tabular}

Table 3. Coefficients in Eqn (12a,b) for DBM

\begin{tabular}{ccccc}
\hline \multirow{2}{*}{ DBM } & \multicolumn{3}{c}{$\mathrm{T}\left[{ }^{\circ} \mathrm{C}\right]$} \\
\cline { 2 - 5 } & & 10 & 20 & 40 \\
\hline \multirow{3}{*}{$1 / c$} & $\mathrm{a}_{c}$ & $1.14 \mathrm{E}-10$ & $9.70 \mathrm{E}-10$ & $5.95 \mathrm{E}-09$ \\
\cline { 2 - 5 } & $\mathrm{b}_{c}$ & $1.12 \mathrm{E}-25$ & $3.35 \mathrm{E}-22$ & $2.50 \mathrm{E}-16$ \\
\cline { 2 - 5 } & $\beta$ & 5 & 4.5 & 3 \\
\hline \multirow{2}{*}{$\mathrm{C}_{\alpha}$} & $\mathrm{a}_{c}$ & 309.0 & 251.6 & 35.3 \\
\cline { 2 - 5 } & $\mathrm{b}_{c}$ & $6.43 \mathrm{E}-06$ & $1.00 \mathrm{E}-04$ & $4.30 \mathrm{E}-03$ \\
\cline { 2 - 5 } & $\gamma$ & 2.5 & 2 & 1.5 \\
\hline
\end{tabular}

$\varepsilon(t)=\int_{0}^{t} \frac{\sigma(\tau)}{c(\sigma(\tau))} d \tau+\int_{0}^{t} \frac{(t-\tau)^{\alpha(\sigma(\tau))-1} \sigma(\tau)}{C_{\alpha}(\sigma(\tau)) \cdot \Gamma(\alpha(\sigma(\tau)))} d \tau$,

because in $0 \div t$ we suppose that the stress $\sigma(\tau) \equiv \sigma_{0}$, then the corresponding strain is given as:

$$
\varepsilon(t)=\frac{1}{c\left(\sigma_{0}\right)}\left(I_{0^{+}}^{1} \sigma\right)(t)+\frac{1}{C_{\alpha}\left(\sigma_{0}\right)}\left(I_{0^{+}}^{\alpha}\left(\sigma_{0}\right) \sigma\right)(t)
$$

Unfortunately, Eqn (13) remains valid only for constant or monotonic stress history. In order to highlight this concept an experimental creep recovery test was contrasted with the solution obtained from Eqn (13). In Figure 7a we may observe that during the creep phase the results predicted by Eqn (13) perfectly match the experimental curve, but for $t>t^{*}, t^{*}$ being the time of which the load was removed, the result of Eqn (13) is drastically differ- ent from experimental curve during the recovery phase. This means that the hypothesis that the coefficients depend only on $\sigma$ and $T$ is not true when $\sigma<0$. Therefore when the stress history is not monotonic the dependence on the various parameters has to be highlighted outside of the viscoelastic behaviour, that is:

$c=c(T, \sigma, \dot{\sigma}) ; \mathrm{C}_{\alpha}=\mathrm{C}_{\alpha}(T, \sigma, \dot{\sigma}) ; \alpha=\alpha(T, \sigma, \dot{\sigma}) \cdot(14 \mathrm{a}, \mathrm{b}, \mathrm{c})$

Moreover, the dependence of the parameters on $\dot{\sigma}$ has to be neglected if $\dot{\sigma}>0$. In order to achieve this result the function sign $\dot{\sigma}$ will present what it happens in plasticity. It follows that the new attempt for modelling the coefficient is:

$c(T, \sigma, \dot{\sigma})=c^{(1)}(T, \sigma)+\operatorname{sign}(\tilde{\mathrm{A}}) c^{(2)}(T, \sigma) ;$

$C_{\alpha}(T, \sigma, \dot{\sigma})=C_{\alpha}^{(1)}(T, \sigma)+\operatorname{sign}(\dot{\sigma}) C_{\alpha}^{(2)}(T, \sigma) ;(15 \mathrm{a}, \mathrm{b}, \mathrm{c})$

$\alpha(T, \sigma, \dot{\sigma})=\alpha^{(1)}(T, \sigma)+\operatorname{sign}(\dot{\sigma}) \alpha^{(2)}(T, \sigma)$

under the conditions that in the creep phase:

$c^{c}(T, \sigma)=c^{(1)}(T, \sigma)+c^{(2)}(T, \sigma) ;$

$C_{\alpha}{ }^{c}(T, \sigma)=C_{\alpha}{ }^{(1)}(T, \sigma)+C_{\alpha}{ }^{(2)}(T, \sigma) ;$

$(16 a, b, c)$

$\alpha^{c}(T, \sigma)=\alpha^{(1)}(T, \sigma)+\alpha^{(2)}(T, \sigma)$,

where the apex " $c$ " stands for creep in the sense that they are already evaluated during the creep test.

These conditions guarantee that if the load history is monotonic then we came back to Eqns (10)-(13), whereas when $\dot{\sigma}<0(\operatorname{sign}(\dot{\sigma})=-1)$ the various parameters change. In order to identify the quantities, $c^{(1)}, c^{(2)}$, $C_{\alpha}{ }^{(1)}, C_{\alpha}{ }^{(2)}, \alpha^{(1)}, \alpha^{(2)}$ we need other conditions that may be derived from creep recovery test. When the recovery phase at time $t^{*}$ at which the stress is removed $(\dot{\sigma}=-\infty$, $\operatorname{sign}(\dot{\sigma})=-1)$ we may identify the new set of parameters $c^{r}(T, \sigma), C_{\alpha}^{r}(T, \sigma)$ and $\alpha^{r}(T, \sigma)$, where the apex " $r$ " stands for recovery, with a best fitting performed in $\left(t^{*} \div \infty\right)$. For the creep recovery test depicted in Figure 7 and for many other tests not reported here for brevity, the parameters significantly depend on $\dot{\sigma}$ are $c$ and $C_{\alpha}$. For the test in Figure $7 \mathrm{~b}$ they assumed the following values:

$$
\begin{aligned}
& c^{r}\left(1 \mathrm{MP} \alpha, 20{ }^{\circ} \mathrm{C}\right)=9140 \mathrm{MPa} s \\
& C_{\alpha}{ }^{r}\left(1 \mathrm{MPa}, 20{ }^{\circ} \mathrm{C}\right)=920 \mathrm{MPa} s^{\alpha} .
\end{aligned}
$$

Once the recovery coefficient is found from experimental data the values of $c^{(1)}, c^{(2)}, C_{\alpha}^{(1)}$ and $C_{\alpha}^{(2)}$ in Eqn $(15 \mathrm{a}, \mathrm{b})$ may be derived in the form:

$$
\begin{gathered}
C^{(2)}=\frac{c^{c}-c^{r}}{2} ; C^{(1)}=\frac{c^{c}-c^{r}}{2} ; \\
C_{\alpha}{ }^{(2)}=\frac{C_{\alpha}{ }^{c}-C_{\alpha}{ }^{r}}{2} ; C_{\alpha}{ }^{(1)}=\frac{C_{\alpha}{ }^{c}-C_{\alpha}{ }^{r}}{2} ; \\
\alpha^{(2)}=\frac{\alpha^{c}-\alpha^{r}}{2} ; \alpha^{(1)}=\frac{\alpha^{c}-\alpha^{r}}{2} .
\end{gathered}
$$

In Figure $7 \mathrm{~b}$ the deformation history evaluated with Eqn (13) in which $c(T, \sigma, \dot{\sigma})$ and $C_{\alpha}(T, \sigma, \dot{\sigma})$ are evaluated as in 

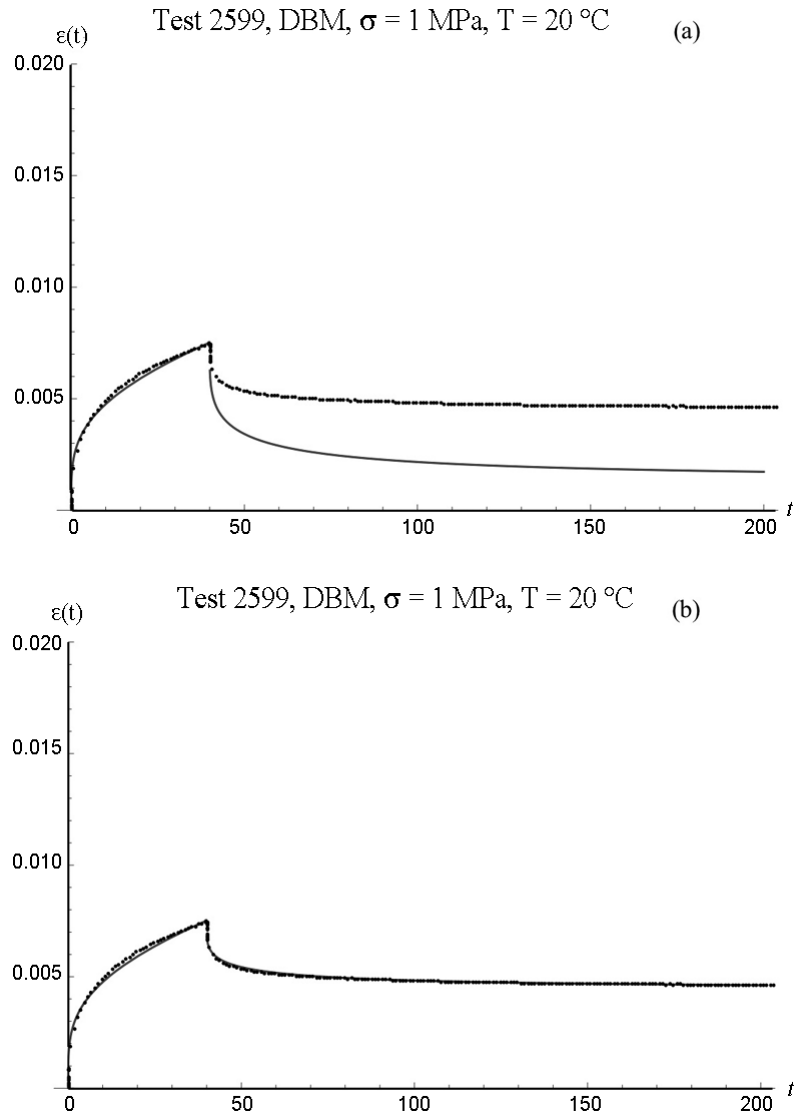

Fig. 7. Creep Recovery test (2599): a) parameters evaluated by Eqn $(11) ;$ b) parameters evaluated by Eqns $(16,18)$

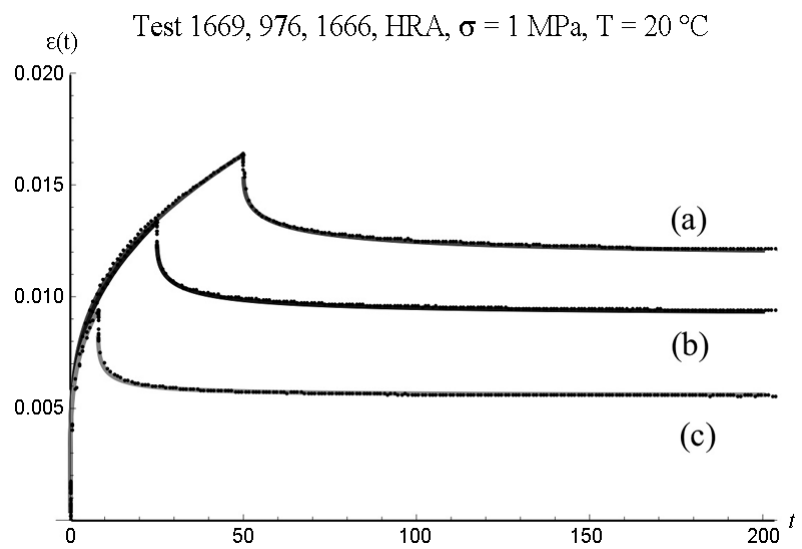

Fig. 8. Experimental curves matched with theoretical laws for creep recovery tests carried out at different loading time: $t^{*}=$ $50 s(\mathrm{a}) ; t^{*}=25 s(\mathrm{~b}) ; t^{*}=8 s(\mathrm{c})$
Eqn $(15 \mathrm{a}, \mathrm{b})$ while $c^{(1)}, c^{(2)}, C_{\alpha}^{(1)}, C_{\alpha}^{(2)}$ and $C_{\alpha}^{(2)}$ as in Eqn $(17 \mathrm{a}, \mathrm{b})$, is contrasted with the experimental creep recovery test.

Other creep recovery tests carried out in Nottingham were fitted using these equations. In Figure 8 three creep recovery tests carried out on the same stress level and temperature and different loading time $t^{*}$ are depicted; in Table 4 the respective relevant parameters are reported.

From Figure 8 some considerations may be drawn. First of all the residual strain at $t \rightarrow \infty$ is evidenced; this fact is easily explained by observing the mechanical model described in Figure 3. During the creep phase the total deformation is composed by two terms: the first one is $\varepsilon_{s}(t)$, that is the deformation of the springpot; the second one is $\varepsilon(t)-\varepsilon_{s}(t)$, that is the relative strain due to the viscous fluid in the dashpot. The latter cannot be given back during the recovery phase, and that confirms the goodness of the mechanical model here presented. A second observation is: the longer the $t^{*}$, at a parity of stress level and temperature, the higher the residual strain. This is due to the fact that during the creep phase the dashpot move according to the equation:

$$
\sigma(t)=c\left[\dot{\varepsilon}(t)-\dot{\varepsilon}_{s}(t)\right]
$$

and $\varepsilon\left(t^{*}\right)-\varepsilon_{s}\left(t^{*}\right)$ is the residual strain that cannot be given back during the recovery phase.

With these observations in mind we may solve separately the two equilibrium equations during the creep phase:

$$
\varepsilon_{s}(t)=\frac{1}{C_{\alpha}\left(\sigma_{0}\right)}\left(I_{0^{+}}^{\alpha} \sigma\right)(t),
$$

where $\sigma(t)=\sigma_{0} U(t)$ and

$$
\varepsilon(t)-\varepsilon_{s}(t)=\frac{1}{c} \sigma_{0} U(t) .
$$

In order to fully validate the model here proposed another test campaign will be necessary with more complex time histories (cyclic stress controlled).

\section{Conclusions}

In this paper a proper mechanical model of hereditariness on asphalt mixtures is presented. It is shown that the creep test, performed on two different asphalt mixtures at various stress levels and temperatures, is

\begin{tabular}{|c|c|c|c|c|c|c|c|c|c|}
\hline \multirow{3}{*}{ Test } & \multirow{3}{*}{$\begin{array}{l}\text { Asphalt } \\
\text { Mixture }\end{array}$} & \multirow{2}{*}{$\begin{array}{c}\text { Air } \\
\text { Voids }\end{array}$} & \multirow{2}{*}{ Stress } & \multirow{2}{*}{$t 1$} & \multirow{2}{*}{$t^{*}$} & \multirow{2}{*}{$T$} & \multicolumn{3}{|c|}{ Fractional proposed model } \\
\hline & & & & & & & $c^{r}$ & $C \alpha^{r}$ & $\alpha$ \\
\hline & & {$[\%]$} & {$[\mathrm{kPa}]$} & {$[\mathrm{s}]$} & {$[\mathrm{s}]$} & {$\left[{ }^{\circ} \mathrm{C}\right]$} & [Mpa s] & {$\left[\mathrm{Mpa} \mathrm{s}^{\alpha}\right]$} & {$[-]$} \\
\hline 1669 & HRA & 5.3 & 1000 & 0.06 & 50 & 20 & 4280 & 529.0 & 0.215 \\
\hline 976 & HRA & 4.8 & 1000 & 0.06 & 25 & 20 & 2735 & 492.0 & 0.215 \\
\hline 1666 & HRA & 5.7 & 1000 & 0.04 & 8 & 20 & 1444 & 423.0 & 0.215 \\
\hline
\end{tabular}
overlapped by the response of a mechanical model

Table 4. Best fitting parameters (coefficients of Eqn 17) of 1669, 976 and 1666 creep recovery tests 
composed by a Maxwell element in which the spring is substituted by a fractional element (springpot). The constitutive law of the springpot is the RiemannLiouville fractional integral of order $\alpha \in(0,1)$. That is the element exhibits an intermediate behaviour between elastic $(\alpha=0)$ and purely viscous Newtonian fluid $(\alpha=1)$.

The dependence of the three different parameters necessary for the definition of the constitutive law on the temperature and on the stress level is studied in detail so obtaining the nonlinear constitutive laws for the asphalt mixture. By the light of the experimental tests it has been observed that, in order to match the experimental data on the recovery phase $(\sigma=0)$, the parameters $c$ and $C_{\alpha}$ have to be split in two parts. In the second part the dependence on has to be present. This part is very important in order to get a residual strain when the stress is removed.

For practical applications the parameter $\alpha$ ruling the power law trend of springpot may be assumed depending only on the temperature and this drastically simplifies the subsequent analysis since in the constitutive law the fractional term remains a simple linear (fractional) operator.

As a concluding remark, in the authors' opinion the correct way to define the proper constitutive law of a complex material like an asphalt mixture has to be validated by experimental tests. The latter have be performed at various temperature levels and at different stress intensity, starting from very small stress level in order to properly define the linear behaviour. Moreover in case of cyclic load history the only creep (or relaxation) test is not enough to fully characterize the viscoplastic behaviour.

Other models, as the Huet-Sayegh model or the most recent 2S2P1D (Olard, Di Benedetto 2003) developed by Di Benedetto, have obtained great results especially in describing the response at cyclic loading for bituminous materials, using six mechanical parameters and seven ones respectively. The second model was also performed on aged and unaged bituminous binder (Yusoff et al. 2013) while the first one was applied into a large number of researches facing both bitumens and mixes.

\section{References}

Airey, G. D.; Rahimzadeh, B.; Collop, A. C. 2003. Viscoelastic linearity limits for bituminous materials, Materials and Structures 36(10): 643-647. http://dx.doi.org/10.1007/BF02479495

Airey, G. D.; Rahimzadeh, B. 2004. Combined bituminous binder and mixture linear rheological properties, Construction and Building Materials 18(7): 535-548. http://dx.doi.org/10.1016/j.conbuildmat.2004.04.008

Bagley, R. L.; Torvik, P. J. 1983. A theoretical basis for the application of fractional calculus to viscoelasticity, Journal of Rheology 27(3): 201-210.

http://dx.doi.org/10.1122/1.549724
Bagley, R. L.; Torvik, P. J. 1986. On fractional calculus model of viscoelastic behaviour, Journal of Rheology 30(1): 133-55. http://dx.doi.org/10.1122/1.549887

British Standards Institution. 2003a. Hot rolled asphalt for roads and other paved areas. BS 594: Part 1. London.

British Standards Institution. 2003b. Coated macadams for roads and other paved areas. BS 4987: Part 1. London.

Celauro, C.; Fecarotti, C.; Pirrotta, A.; Collop, A. C. 2012. Experimental validation of a fractional model for asphalt mixtures, Construction and Building Materials 36: $458-466$ http://dx.doi.org/10.1016/j.conbuildmat.2012.04.028

Christensen, R. M. 1982. Theory of viscoelasticity an introduction. London: Academic Press. 378 p.

Di Paola, M.; Pirrotta, A.; Celauro, C.; Lo Presti, D.; Marino, F. 2009. Modeling of the viscoelastic behavior of paving bitumen using fractional derivates, Meccanica dei Materiali e delle Strutture I (II): 38-51.

Di Paola, M.; Pirrotta, A.; Valenza, A. 2011. Visco-elastic behavior through fractional calculus: an easier method for best fitting experimental results, Mechanics of Materials 43(12): 799-806. http://dx.doi.org/10.1016/j.mechmat.2011.08.016

Di Paola, M.; Zingales, M. 2012. Exact mechanical models of fractional hereditary materials, Journal of Rheology 9(5): 983-1004. http://dx.doi.org/10.1122/1.4717492

Di Paola, M.; Pinnola, F. P.; Zingales, M. 2013a. A discrete mechanical model of fractional hereditary materials, Meccanica, 1-14. http://dx.doi.org/10.1007/s11012-012-9685-4

Di Paola, M.; Pinnola, F. P.; Zingales M. 2013b. Fractional differential equations and related exact mechanical models, Computers and Mathematics with Applications 66(5 September): 608-620. http://dx.doi.org/10.1016/j.camwa.2013.03.012

Findley, W. N.; Onaran, K. 1976. Creep and relaxation of nonlinear viscoelastic materials. Dover Civil and Mechanical Engineering. Amsterdam, New York, Oxford: North Holland Publishing Company. 371 p.

Flügge, W. 1976. Viscoelasticity. Berlin, Heidelberg, New York: Springer-Verlag. $194 \mathrm{p}$.

Grzesikiewicz, W.; Wakulicz, A.; Zbiciak, A. 2013. Nonlinear problems of fractional calculus in modelling of mechanical systems, International Journal of Mechanical Science 70: 90-98. http://dx.doi.org/10.1016/j.ijmecsci.2013.02.007

Huang, Y. H. 2004. Pavement analysis and design. NJ, Englewood Cliffs: Prentice-Hall. 775 p.

Koeller, R. C. 1984. Application of fractional calculus to the theory of viscoelasticity, Journal of Applied Mechanics 51(2): 299-307. http://dx.doi.org/10.4236/am.2011.212214

Liu, Y.; You, Z. 2009. Determining burger's model parameters of asphalt materials using creep-recovery testing data, Pavements and Materials, 26-36. http://dx.doi.org/10.1061/41008(334)3

Mainardi, F. 2010. Fractional calculus and waves in linear viscoelasticity. London: Imperial College Press, World Scientific. 368 p.

Nutting, P. G. 1921. A new general law of deformation, Journal of the Franklin Institute 191: 679-685.

Oeser, M.; Pellinen, T.; Scarpas, A.; Kasbergen, C. 2008. Studies on creep and recovery of rheological bodies based upon conventional and fractional formulations and their application on asphalt mixture, International Journal of Pavement Engineering 9(5): 373-386. http://dx.doi.org/10.1080/10298430802068923

Olard, F.; Di Benedetto, H. 2003. General "2S2P1D” model and relation between the linear viscoelastic behaviours of 
bituminous binders and mixes, Road Materials and Pavement Design 4(2): 185-224.

http://dx.doi.org/10.1080/14680629.2003.9689946

Podlubny, I. 1999. Fractional differential equations. Mathematics in science and engineering, Vol. 198. San Diego: Academic Press.

Slonimsky, G. L. 1967. Laws of mechanical relaxation processes in polymers, Journal of Polymer Science 16: 1667-1672. http://dx.doi.org/10.1002/polc.5070160342

Smit, W.; de Vrie, H. 1970. Rheological models containing fractional derivatives, Rheologica Acta 9: 525-34. http://dx.doi.org/10.1007/BF01985463

Soczkiewicz, E. 2002. Application of fractional calculus in the theory of viscoelasticity, Molecular and Quantum Acoustics 23: 397-404.

Stastna, J.; Zanzotto, L.; Ho, K. 1994 Fractional complex modulus manifested in asphalts, Rheologica Acta 33(4): 344-54. http://dx.doi.org/10.1007/BF00366961

UNI EN 12697-25. 2005. Bituminous mixtures - Test methods for hot mix asphalt - Part 25: Cyclic compression test. Brussels.
Taherkhania, H. 2011. Compressive creep behaviour of asphalt mixtures, Procedia Engineering 10: 583-588. http://dx.doi.org/10.1016/j.proeng.2011.04.097

Werkmeister, S. et al. 2013. Re-road - end of strategies of asphalt pavements. Deliverable D 5.3. model development and validation. European Commission DG Research, A FP7 Collaborative Project.

Yusoff, Md.; Mounier, D.; Airey, G. D. 2013. The 2S2P1D: an exellent linear viscoelastic model, UNIMAS e-Journal of Civil Engineering.

Zanzotto, L.; Stastna, J.; Ho, K. 1996 Characterization of regular and modified bitumens via their complex modulus, Journal of Applied Polymer Science 59(12): 1897-1905. http://dx.doi.org/10.1002/(SICI)1097-4628(19960321)59: $12<1897:: A I D-A P P 12>3.0 . C O ; 2-P$

Zbiciak, A. 2013. Mathematical description of rheological properties of asphalt-aggregate mixes, Bulletin of the Polish Academy of Sciences 61(1): 65-72. http://dx.doi.org/10.2478/bpasts-2013-0005

Gaetano DI MINO. He is an Associate Professor at the Faculty of Engineering, University of Palermo. He is author of about 60 publications. His research activity is mainly focused on: the mechanical behaviour of bituminous mixtures; the mix-design of porous asphalt; the FEM modelling of ground borne vibration from railway traffic; the approach based on the genetic algorithm for the road superstructure's maintenance and rehabilitation.

Gordon AIREY. He is a Professor of Pavement Engineering Materials and Director of the Nottingham Transportation Engineering Centre (NTEC) at the University of Nottingham. In addition, he is Deputy Head of the Division of Infrastructure, Geomatics and Architecture within the Faculty of Engineering, a Director on the Board of the International Society for Asphalt Pavements (ISAP) and member of the ISAP Executive Committee. His current research interests include fundamental rheological testing of bitumen and mastics; the durability of asphalt materials; and the use of physicochemical procedures to address moisture damage and adhesion loss in asphalt mixtures.

Mario DI PAOLA. He is a Full Professor, teaching Dynamics of Structures, at the Faculty of Engineering, University of Palermo. He is author of about 250 publications of which more than 115 on international journals and 7 parts of scientific books. The research activity is mainly focused on: random dynamics, safety assessment of structures in seismic areas, stochastic differential calculus, structures exposed to wind and raging sea, identification of damage, random parameters structures, modal correction methods, Monte-Carlo simulation, non-local mechanics, fractional calculus and applications.

Francesco Paolo PINNOLA. He carries out the PhD in Structural Engineering under supervision of Prof. Mario Di Paola at University of Palermo and his research topics involve the continuous mechanics with particular regard to fractional viscoelasticity, and stochastic mechanics through the use of fractional calculus to describe random processes and variables. Currently he is a visiting Research Scholar at the Department of Civil and Environmental Engineering of Rice University, Houston (TX) and he is studying stochastic mechanics under the guidance of Prof. Pol D. Spanos.

Giacomo D'ANGELO. He received Master's Degree (Laurea Magistralis) in Civil Engineering/Construction Engineering (honors) at University of Palermo in 2013. His research topics are related to transport infrastructure engineering with particular focus to bituminous materials mechanical behaviour, rheology, fractional viscoelasticity, design, maintenance and rehabilitation of road pavements and railways.

Davide LO PRESTI. He has been appointed as a Post-Doctoral Research Fellow at the University of Nottingham where he has mainly worked as co-investigator under the 4M $€$ EU FP7 project "ReRoad- End of Life Strategies of Asphalt Pavements". His research interests include the transport infrastructure engineering with particular focus on the recycling of waste materials into the road pavements, rheology of bituminous binders, bituminous materials development and other sustainable practices for new design, maintenance and rehabilitation of road pavements and railways. 